\title{
Expression of KL-6/MUC1 in pancreatic cancer tissues and its potential involvement in tumor metastasis
}

\author{
HUANLI XU ${ }^{1,2}$, YOSHINORI INAGAKI ${ }^{1}$, YASUJI SEYAMA ${ }^{1}$, GUANHUA DU ${ }^{2}$, \\ FENGSHAN WANG $^{3}$, NORIHIRO KOKUDO ${ }^{1}$ and WEI TANG ${ }^{1}$
}

\begin{abstract}
${ }^{1}$ Hepato-Biliary-Pancreatic Surgery Division, Department of Surgery, Graduate School of Medicine, University of Tokyo, Tokyo 113-8655, Japan; ${ }^{2}$ Institute of Materia Medica, Chinese Academy of Medical Sciences, Beijing 100050; ${ }^{3}$ Institute of Biochemical and Biotechnological Drugs, School of Pharmaceutical Sciences, Shandong University, Jinan 250012, Shandong, P.R. China
\end{abstract}

Received January 19, 2011; Accepted March 30, 2011

DOI: 10.3892/or.2011.1315

\begin{abstract}
Aberrant expression of KL-6/MUC1 mucin has been proven to be associated with poorer tumor behavior in many carcinomas. The aim of this study was to evaluate the expression of KL-6/MUC1 in pancreatic cancer tissues and its potential involvement in tumor metastasis. The expression of KL-6/MUC1 in 18 cases of pancreatic ductal carcinoma (PDC), 5 cases of intraductal papillary mucinous tumor (IPMT), and 3 cases of islet cell tumor was detected by immunohistochemical staining. To determine the impact of loss of KL-6/MUC1 expression on pancreatic tumor progression, an siRNA targeting MUC1 was synthesized and transfected into Panc-1 cells and Capan-1 cells to knock down KL-6/MUC1 expression. Down-regulation of KL-6/MUC1 expression was detected by reverse transcription-polymerase chain reaction (RT-PCR) and Western blotting. E-cadherin and KL-6 mucin co-expression was detected by immunofluorescence. The expression of E-cadherin and E-cadherin/ $\beta$-catenin comlex was determined by immunoprecipitation. Cell invasive abilities were detected by invasion assay. Positive KL-6/MUC1 staining was observed in all 18 PDC cases $(18 / 18,100.0 \%)$ and 1 metastatic IPMT case $(1 / 5,20.0 \%)$. The results suggested that KL-6/MUC1 overexpression may be associated with more aggressive tumor behavior, although the cases of pancreatic cancer tissues in this study are limited. RT-PCR and Western blotting showed that both KL-6/MUC1 mRNA and protein can be effectively silenced. Following KL-6/ MUC1 knockdown, E-cadherin expression increased. Also,
\end{abstract}

Correspondence to: Dr Wei Tang, Hepato-Biliary-Pancreatic Surgery Division, Department of Surgery, Graduate School of Medicine, University of Tokyo, Hongo 7-3-1, Tokyo 113-8655, Japan E-mail: tang-sur@h.u-tokyo.ac.jp

Key words: KL-6/MUC1, pancreatic carcinoma, RNA interference, E-cadherin, $\beta$-catenin
E-cadherin/ $\beta$-catenin complex expression increased and the invasive ability of the cells decreased. This study indicated that overexpression of KL-6/MUC1 in pancreatic cancer tissues may be associated with metastasis of pancreatic cancer by regulating E-cadherin and E-cadherin/ $\beta$-catenin complex expression.

\section{Introduction}

In spite of the recent efforts to improve prevention, screening, and therapy, pancreatic cancer has a poor prognosis: a 5-year survival rate of $\sim 6 \%$ and a median survival of $<6$ months (1). The major problem in the management of postsurgical cases is failure to control cancer micrometastases, which results from a lack of early detection and effective treatment. A monoclonal antibody ( $\mathrm{mAb}$ ) with high specificity and affinity could be used for targeted therapy, and may serve to overcome this problem.

MUC1 is a polymorphic, highly glycosylated, type I transmembrane glycoprotein expressed by ductal epithelial cells of secretory organs, including the pancreas, breast, lung, and gastrointestinal tract (2). KL-6/MUC1, a type of MUC1 categorized as cluster 9 , is recognized by KL-6 mAb, and its epitope includes sialo-oligosaccharide moiety in MUC1 molecules (3). This mucin was first established in the serum of patients with intestinal pneumonia (4) but has recently been detected in various cancer tissues. Recent histological and biological studies have suggested that aberrant expression of KL-6/MUC1 in cancer tissues was associated with worse tumor behavior such as invasion and metastasis in ampullary carcinoma (5), primary colorectal carcinoma (6), and cholangiocarcinoma $(7,8)$. Our previous study also showed that positive KL-6/MUC1 expression was associated with invasion of pancreatic cancer cells (9). Little is known about the expression profile of KL-6/MUC1 in pancreatic carcinoma tissues and its functional roles in metastasis.

E-cadherin and its associated proteins, such as $\beta$-catenin, are principal components of intercellular adherens junctions and mediate cell-cell adhesion. In most epithelial cancers, loss of E-cadherin mediated cell-cell adhesion is concomitant 
with tumor progression. Abnormal E-cadherin expression and poor clinical outcome in pancreatic cancer patients has been established (10). E-cadherin loss during tumor progression can be caused by various genetic and epigenetic mechanisms. Some studies have reported that cancer patients with aberrant MUC1, E-cadherin or $\beta$-catenin expression have a worse prognosis when compared to similarly matched patients $(11,12)$. The objective of the current study was to analyze the impact of alterations in KL-6/MUC1 expression on E-cadherin/ $\beta$-catenin expression in pancreatic cancer cells and its effect on cell migration. In this study, we used RNAi methods to suppress KL-6/MUC1 expression in Panc-1 and Capan-1 cells and investigated the impact of decreased KL-6/ MUC1 expression on tumor behavior of pancreatic cancer cells.

\section{Materials and methods}

Materials. Trypsin, regents for cell lysis, trypan blue and culture media were purchased from Sigma Chemical Co., St. Louis, MO, USA; KL-6 mAb was provided by Eisai Co., Ltd., Tokyo, Japan; Histofine SAB-PO kit was pursed from Nichirei Corporation, Tokyo, Japan; ECL Western Blotting Starter Kit was purchased from GE Healthcare Bio-Sciences, Piscataway, NJ, USA; Diff-Quik kit was purchased from Sysmex, Kobe, Japan; Cell Proliferation Kit was purchased from Roche Applied Science Mannheim, Germany; Annexin V-FITC apoptosis detection kit, antiE-cadherin, anti- $\beta$-catenin, anti- $\beta$-actin antibody, BD BioCoat $^{\mathrm{TM}}$ Tumor Invasion System were purchased from BD Pharmingen ${ }^{\mathrm{TM}}$ CA, USA; MUC1 targeting siRNA pool, Non-targeting siRNA pool \#1, siGLO green siRNA, Dharma Fect transfection regent 4, 5x siRNA buffer, molecular grade RNase-free water, were purchased from Thermo Scientific Dharmacon, USA.

Human pancreatic cancer tissues. Human pancreatic ductal carcinoma (PDC) specimens were collected from 18 patients [11 male, 7 female; average age 67.3 years (range 54-82)]. Intraductal papillary mucinous tumor (IPMT) tissues were resected from 5 patients [ 3 male, 2 female; average age 57.8 years (range 42-66)]. Islet cell tumor tissues were collected from 3 patients [0 male, 3 female; average age 46.6 years (range 32-60)]. Patients underwent surgical resection at the Hepato-Biliary-Pancreatic Surgery Division of the Department of Surgery, Graduate School of Medicine, the University of Tokyo between January 1998 and December 2000. Their clinicopathological characteristics were evaluated according to the general rules for the study of pancreatic cancer, which were set by the Japan Pancreas Society $(13,14)$.

Immunohistochemical staining for KL-6/MUC1 in human pancreatic cancer tissues. KL-6/MUC1 expression in various pancreatic cancer tissues was detected by immunohistochemical staining. Briefly, 5- $\mu$ m-thick sections were cut from archival formalin-fixed paraffin-embedded tissue blocks, deparaffinized, and dehydrated through a graded series of ethanol. Endogenous peroxidase activity was halted through administration of $0.3 \%$ hydrogen peroxide/methanol for $30 \mathrm{~min}$. Then sections were blocked with normal goat serum for $30 \mathrm{~min}$ at room temperature, and then incubated with or without KL-6 antibody (1:1000 dilution) for $60 \mathrm{~min}$ at room temperature. After the incubation of biotin-labeled secondary antibody, detection of KL-6/MUC1 was achieved by the biotin-streptavidin-peroxidase complex method using a commercial kit. 3,3'-Diaminobenzidine was used as the chromogen, and haematoxylin was used as a counterstain.

Cells and cell culture conditions. Panc-1 cells (human pancreatic duct carcinoma cell line) were obtained from RIKEN Cell Bank, Ibaraki, Japan. Capan-1 cells (another human pancreatic duct adenocarcinoma cell line) were obtained from American Type Culture Collection, USA. Panc-1 cells were cultured in RPMI-1640 supplemented with $10 \%$ fetal bovine serum (FBS) in a humidified atmosphere with $5 \%$ $\mathrm{CO}_{2}$ in air at $37^{\circ} \mathrm{C}$. Capan-1 cells were cultured in ATCC's Iscove's modified Dulbecco's medium (IMDM) supplemented with $20 \%$ FBS. For maintenance and subculture, Panc-1 cells in exponential phase were treated with $0.02 \%$ EDTA and Capan- 1 cells with $0.25 \%$ trypsin solution containing $0.02 \%$ EDTA. After reaching $80 \%$ of confluence, cells were collected for the following experiments.

Transfection and condition optimization. Panc-1 and Capan-1 cells were transfected with MUC1-specific siRNA (siGenome smartpool), or non-targeting siRNA pool 1\# according to the manufacturer's instructions. siGLO Green transfection indicator was used to determine optimal siRNA transfection conditions. In 96-well plates, the optical transfection conditions for Panc- 1 cells were as follows: $5 \times 10^{3}$ cells/ well, $50 \mathrm{nmol} / 1 \mathrm{siRNA}, 0.2 \mathrm{ml}$ transfection reagent. For Capan-1 cells, the optical transfection conditions were as follows: $5 \times 10^{3}$ cells/well, $100 \mathrm{nmol} / 1 \mathrm{siRNA}, 0.8 \mathrm{ml}$ transfection reagent. Each experiment includes the following samples in triplicate: i) untreated cells; ii) mock-transfection (transfection regent only); iii) MUC1 siRNA; and iv) non-targeting siRNA (negative control siRNA). At specific time points after transfection, cells were fixed immediately for mRNA analysis and immunofluorescence staining, extracted for reverse transcription-polymerase chain reaction (RT-PCR), Western blot analysis and immunoprecipitation, or prepared for cell invasion assay.

RT-PCR for KL-6/MUC1. To determine the effects of KL-6/ MUC1 knockdown, KL-6/MUC1 mRNA levels were determined using real-time RT-PCR. Briefly, RNA was extracted from cells using TRIzol reagent as recommended by the manufacturer. Equal amounts of RNA were converted to cDNA using M-MLV reverse transcriptase. Quantitative RT-PCR using SYBR-Green dye was performed using Thermal Cycler DiceT ${ }^{\mathrm{TM}}$ Real-Time System (TP 800, Takara Bio Inc., Ohtsu, Japan). Primers were as follows: KL-6/ MUC1 forward, 5'-AGACGTCAGCGTGAGTGATG-3', KL-6/MUC1 reverse, 5'-GACAGCCAAGGCAATGAGAT-3', length: $172 \mathrm{bp}$; the GAPDH forward, 5'-TGGTATCG TGGAAGGACTCATGAC-3', GAPDH reverse, 5'-ATGC CAGTGAGCTTCCCGTTCAGC-3', length, 189 bp. The PCR cycling conditions were as follows: initial denaturation at $95^{\circ} \mathrm{C}$ for $30 \mathrm{sec}, 33$ cycles of denaturation at $95^{\circ} \mathrm{C}$ for $15 \mathrm{sec}$, annealing at $55^{\circ} \mathrm{C}$ for $20 \mathrm{sec}$, and extension $72^{\circ} \mathrm{C}$ for $20 \mathrm{sec}$, 
followed by a final extension of $95^{\circ} \mathrm{C} 15 \mathrm{sec}, 60^{\circ} \mathrm{C} 30 \mathrm{sec}, 95^{\circ} \mathrm{C}$ $15 \mathrm{sec}$. The relative gene expression was determined using the

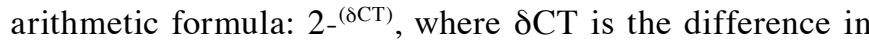
CT value between the gene of interest and the housekeeping gene, GAPDH. All quantitative RT-PCR experiments were performed in duplicate and repeated four times. Statistical analysis of these measurements consisted of an independent t-test. The mean difference was considered significant at the 0.05 level for all data $(\mathrm{P}<0.05)$.

Western blotting. After transfection for $48 \mathrm{~h}$, cell lysates were prepared using RIPA lysis buffer. Protein concentrations were quantified using a DC Protein Assay. Equal amounts of protein $(20 \mu \mathrm{g})$ were separated by sodium dodecyl sulfate polyacrylamide gel electrophoresis and then electrotransferred onto polyvinylidene difluoride membranes. After blocking with TBST buffer (20 mM Tris-buffered saline and $0.1 \%$ Tween-20) containing $5 \%$ BSA overnight at $4{ }^{\circ} \mathrm{C}$, the membranes were incubated with different antibodies (anti-KL-6/MUC1 antibody, 1:750 dilution; anti-E-cadherin antibody, 1:1000; anti- $\beta$-catenin antibody, 1:1000; anti- $\beta$-actin antibody, 1:1000 dilution) for $1 \mathrm{~h}$, which was followed by washing for 3 times. After incubation with HRP-conjugated secondary antibodies for $1 \mathrm{~h}$ at room temperature, the reactivity was visualized by enhanced chemiluminescence using ECL Western Blotting Starter Kit.

Immunoprecipitation. Immunoprecipitation assay was performed to detect the expression of E-cadherin/ $\beta$-catenin complex in the following manner. Cells were lysed in RIPA buffer and then cell lysates were incubated with E-cadherin antibody (1:200 dilution) for $2 \mathrm{~h}$ at $4{ }^{\circ} \mathrm{C}$. Immunoprecipitates were prepared by incubation overnight with protein $\mathrm{G}$ agarose beads (KPL, Inc., Gaithersburg MD, USA). Pellets were washed three times with RIPA and analyzed by Western blotting.

Immunofluorescence staining. Immunofluorescence staining was used to detect the expression of KL-6/MUC1 and E-cadherin. Briefly, $48 \mathrm{~h}$ after transfection, cells were fixed with $3.75 \%$ formaldehyde for $10 \mathrm{~min}$, rinsed and permeabilized with $0.2 \%$ Triton solution for $30 \mathrm{~min}$. After blocking, the cells were incubated with a mixture of KL-6 antibody (1:200) and E-cadherin antibody (1:200) overnight at $4^{\circ} \mathrm{C}$. After rinsing, the cells were incubated at $37^{\circ} \mathrm{C}$ for $1 \mathrm{~h}$ with diluted secondary antibody (anti-mouse IgG1-FITC and anti-mouse IgG2a-PE, 1:200). Then the cells were fixed and covered with mount medium and DAPI. Fluorescence images were obtained using a fluorescence microscope (model BZ-9000; Keyence, Osaka, Japan).

Transwell chamber assay. After transfection, a suspension of cells $\left(2 \times 10^{5}\right.$ cells $\left./ 100 \mu \mathrm{l}\right)$ was placed in the control and matrigel-coated filters. The wells below were filled with $0.75 \mathrm{ml}$ RPMI-1640 medium supplemented with 5\% FBS. Cells were allowed to migrate for $22 \mathrm{~h}$ at $37^{\circ} \mathrm{C}$. Migration was terminated by removing the cells from the upper compartment of the filter with a cotton swab. Cells that had invaded through and reached the lower surface of filters were stained by Diff-Quik kit. The migrated cells were quantified by
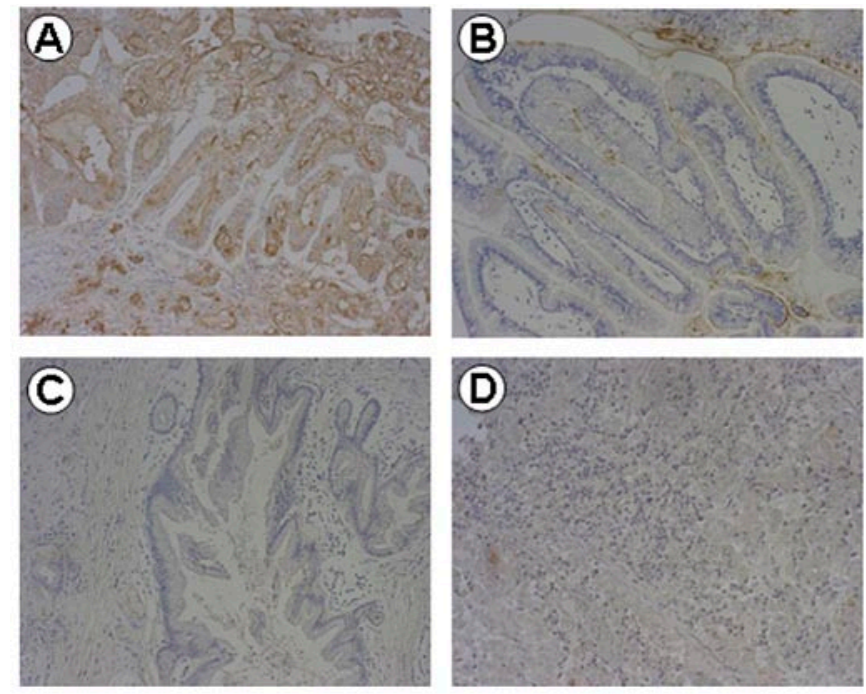

Figure 1. KL-6/MUC1 Expression in various pancreatic cancer tissues. (A) positive KL-6/MUC1 expression in PDC tissues; (B) positive KL-6/MUC1 expression in IPMTs tissues; (C) negative KL-6/MUC1 expression in IPMTs tissues; (D) negative KL-6/MUC1 expression in islet cell tumor tissues; original magnification, $\mathrm{x} 200$.

counting the number of cells in eight random microscopic fields per filter at a magnification of $\times 200$. Statistical significance was determined by Student's two-tailed t-test. The limit of statistical significance was $\mathrm{P}<0.05$.

\section{Results}

KL-6/MUC1 expression in human pancreatic cancer tissues. Immunohistochemical analysis was performed to determine the expression characteristics of KL-6/MUC1 in PDC, IPMT, and islet cell tumor tissues used in this study. All of the 18 cases of PDC showed positive staining for KL-6/MUC1. Tumor cell cytoplasm and luminal surfaces, as well as the inner surfaces of glands in cancer regions, were strongly stained (Fig. 1A). The population of KL-6/MUC1 positive tumor cells in the cancer regions varied among the samples (mean $83.6 \%$, range 57.0-95.0\%). Positive staining was rarely observed in the surrounding normal pancreatic tissues (data not shown). In all the 18 cases of PDC, venous invasion, lymphatic invasion, as well as liver or lymph node metastasis were found.

Positive staining can be observed only in the inner surfaces of glands in 1 case of the IPMT tissues $(1 / 5,20.0 \%)$, in which case lymph node metastasis was found (Fig. 1B). Positive KL-6/MUC1 staining was not found in the islet cell tumor tissues (Fig. 1D).

Effect of siRNA transfection on KL-6/MUC1 mRNA levels. RT-PCR showed that KL-6/MUC1 mRNA can be effectively silenced after transfection in optimal transfection conditions. The inhibition rates of MUC1 siRNA on Panc-1 cell KL-6/ MUC1 mRNA were 85.03, 81.78, and 75.09\% after 24, 48, and $72 \mathrm{~h}$ transfection, respectively (Fig. 2A). The inhibition rates of MUC1 siRNA on Capan-1 cell KL-6/MUC1 mRNA were 56.7, 58.1, and $45.8 \%$ after 24,48 and $72 \mathrm{~h}$ transfection, respectively (Fig. 2B). Additionally, no change was found in 
A
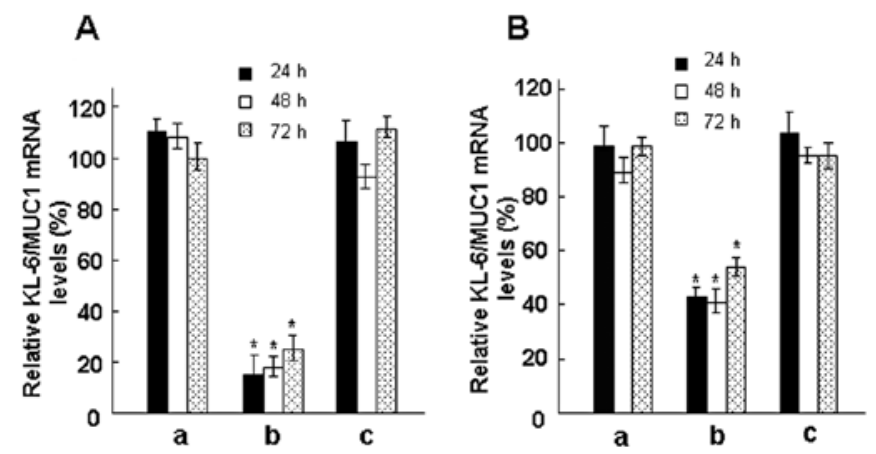

Figure 2. Relative KL-6/MUC1 mRNA levels in Panc-1 (A) and Capan-1 cells (B) by RT-PCR. (a) Tansfection reagent only; (b) MUC1 siRNA; (c) non-targeting siRNA for 24,48 and $72 \mathrm{~h}$. ${ }^{*} \mathrm{P}<0.05$.

\section{A}

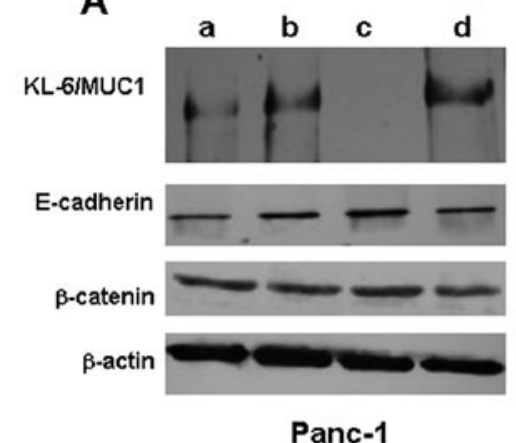

B

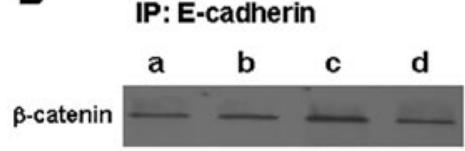

Panc-1
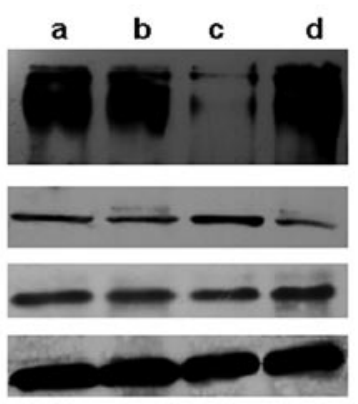

Capan-1

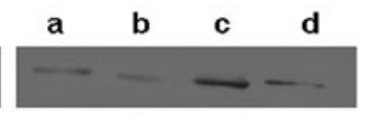

Capan-1

Figure 3. KL-6/MUC1, E-cadherin, $\beta$-catenin (A) and E-cadherin/ $\beta$-catenin complex (B) expressions in Panc-1 and Capan-1 cells. (a) Control; (b) transfection reagent only; (c) MUC1 siRNA; (d) non-targeting siRNA.

KL-6/MUC1 mRNA levels in the cells transfected by transfection regent only and non-targeting siRNA compared to the untreated cells $(\mathrm{P}>0.05)$.

Effect of siRNA transfection on $K L-6 / M U C 1$, E-cadherin and $\beta$-catenin expressions, and $E$-cadherin/ $\beta$-catenin complex formation. The effect of siRNA transfection on KL-6/MUC1 levels was determined by Western blotting. Fig. 3A demonstrates that KL-6/MUC1 expression was greatly reduced in both Panc-1 and Capan-1 cells incubated with MUC1 siRNA, while no change was found in KL-6/MUC1 expression levels in the cells transfected by transfection reagent only and nontargeting siRNA compared to the untreated cells. Western blotting result also showed that the E-cadherin protein levels were inversely related to KL-6/MUC1 expression. Low levels of E-cadherin protein were observed in Panc-1 cells and their control cell lines while relatively more E-cadherin was observed in cells transfected with KL-6/MUC1 siRNA. Similar data were observed in Capan-1 cells (Fig. 3A). Changes of $\beta$-catenin expression levels were not obvious

A

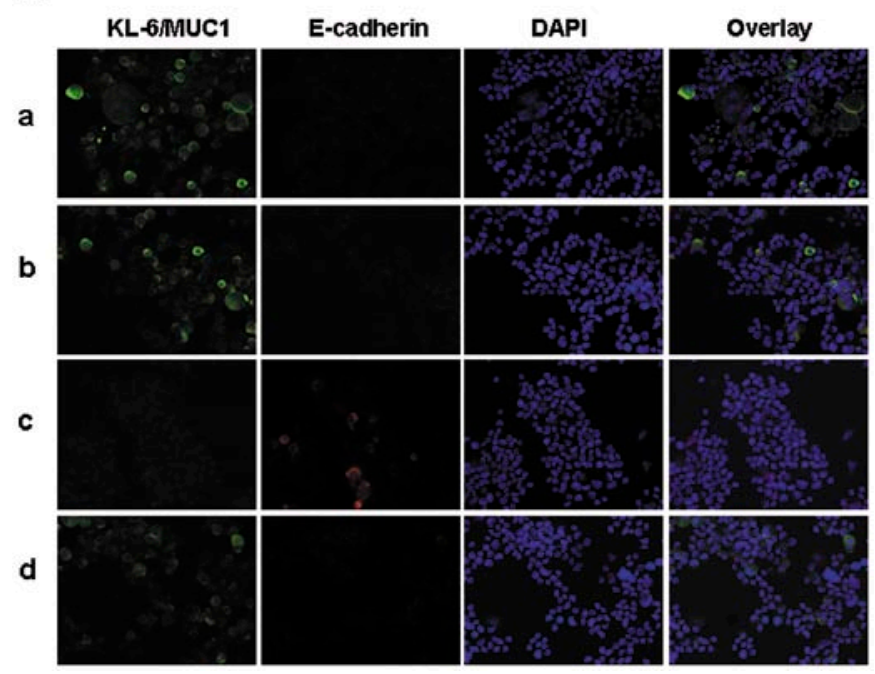

B



Figure 4 (A) KL-6/MUC1 and E-cadherin expression in Panc-1 cells by immunofluorescence staining. (a) Control; (b) transfection reagent only; (c) MUC1 siRNA; (d) non-targeting siRNA. (B) KL-6/MUC1 and E-cadherin expression in Capan-1 cells by immunofluorescence staining. (a) Control; (b) transfection reagent only; (c) MUC1 siRNA; (d) non-targeting siRNA.

after KL-6/MUC1 down-regulation in both cell lines. These data suggested that KL-6/MUC1 might inhibit E-cadherin protein expression by an unknown mechanism.

Immunoprecipitation studies were performed to determine if the increased E-cadherin in Panc-1 and Capan-1 cells with KL-6/MUC1 'knockdown' was complexed to $\beta$-catenin. The result showed that E-cadherin/ $\beta$-catenin complex of cells transfected with MUC1 siRNA was obviously increased compared with that of the control groups (Fig. 3B), which meant that the increased E-cadherin in Panc-1 and Capan-1 cells with KL-6/MUC1 'knockdown' was complexed to $\beta$-catenin.

Effects of siRNA transfection on KL-6/MUC1 and E-cadherin expression. Fig. 4 demonstrates KL-6/MUC1 and E-cadherin expressions in optimal transfection conditions in both Panc-1 


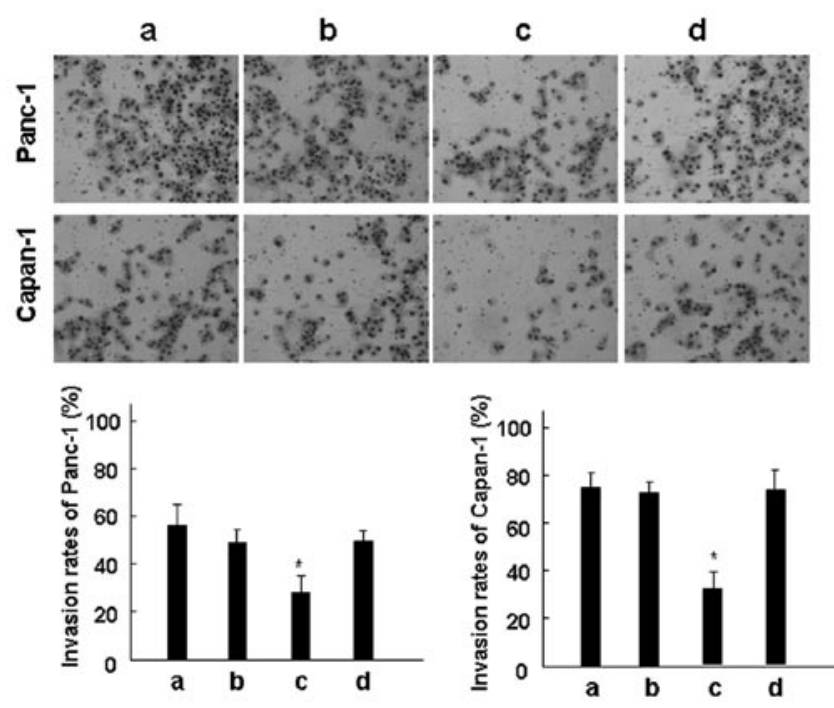

Figure 5. Invasion abilty of Panc-1 and Capan-1 cells by transwell chamber assay. (A) Control; (B) transfection reagent only; (C) MUC1 siRNA; (D) non-targeting siRNA. ${ }^{*} \mathrm{P}<0.05$.

and Capan-1 cells by immunofluorescence staining. Fig. 4A (a, b, and d) and B (a, b, and d) show strong staining for KL-6/MUC1 in both Panc-1 and Capan-1 cells, indicating no change in KL-6/MUC1 protein levels in the untreated cells, cells transfected by transfection reagent only, and cells transfected by non-targeting siRNA. Staining for KL-6/ MUC1 expression was obviously reduced in cells transfected with MUC1 siRNA (Fig. 4Ac and Bc), which confirmed the result of Western blotting. Staining with E-cadherin showed that there were more E-cadherin expressions in both Panc-1 and Capan-1 cells transfected with MUC1 siRNA (Fig. 4Ac and $\mathrm{Bc}$ ), while no change was found in the untreated cells, cells transfected by transfection reagent only, and cells transfected by non-targeting siRNA (Fig. 4A (a, b, and d) and $B$ (a, b, and d).

Effect of siRNA transfection on cell invasion. Since both KL-6/MUC1 overexpression and loss of E-cadherin function have been implicated as mechanisms for cell migration, the impact of decreased KL-6/MUC1 expression on cell invasion was evaluated using a matrigel invasion assay. As shown in Fig. 5, the number of Panc-1 and Capan-1 cells that invaded through matrigel the chamber was significantly less than that observed in control groups $(\mathrm{P}<0.05)$. In Panc-1 cells, the invaded cells per field decreased up to $50.1 \%$ compared with the untreated cells. In Capan-1 cells, the invaded cells per field decreased up to $61.7 \%$ compared with the untreated cells. There was no statistically significant difference in invaded cells transfected by transfection reagent only and non-targeting siRNA compared to the untreated cells $(\mathrm{P}>0.05)$.

\section{Discussion}

PDC is an extremely aggressive malignancy, which progresses rapidly to develop metastatic lesions and carries a dismal prognosis. Control of micrometastatic pancreatic cancer remains a major objective in PDC treatment. IPMTs, which were firstly reported in 1982 by Ohhashi et al as mucinproducing pancreatic cancers, have been increasing in the West and in Japan (15). IPMT is a slowly growing tumor with rare stromal invasion and a good prognosis (16). Islet cell tumor is an uncommon tumor of the pancreas that arises from a type of cell called the islet cell in the pancreas (17). An islet cell tumor is a mass of abnormal cells that forms in the endocrine (hormone-producing) tissues of the pancreas. Islet cell tumors may be benign (non-cancerous) or malignant (cancerous) (17). In the present study, we first demonstrated the expression of the MUC1 antigen in various pancreatic cancer specimens using KL-6 mAb. We found that all the PDC cases showed positive staining for KL-6/MUC1, while the majority of normal pancreas cases did not. Positive KL-6/ MUC1 staining was observed only in the inner surfaces of glands in 1 case of the IPMT tissue $(1 / 5,20 \%)$ (Fig. 1B). Positive staining was not found in tumor cells of any IPMTs or islet cell tumor cases (Fig. 1C and D). This result indicated that overexpression of KL-6/MUC1 in pancreatic cancer tissues may be associated with more aggressive tumor behavior or worse tumor staging.

The functional effect of decreased KL-6/MUC1 in cancer cells was evaluated by in vitro cell migration assays. Decreased KL-6/MUC1 expression in both Panc-1 and Capan-1 cells resulted in decreased migration as measured by a matrigel invasion assay. Several mechanisms have been implicated in KL-6/MUC1 mediated cell migration. Its large, heavily negative charged extra-cellular subunit may directly interfere with cell-cell adhesion. Alternatively, its cytoplasmic tail could affect signal transduction since it can bind to epidermal growth factor receptor (EGFR) family members (18) and c-Src (19). In this study, KL-6/MUC1 regulated expression of the cell adhesion molecule, E-cadherin, which might be another potential mechanism by which KL-6/MUC1 inhibits cell migration. The result showed that reduced KL-6/MUC1 expression, increased E-cadherin expression and restoration of the E-cadherin/ $\beta$-catenin complex may altogether reduce cell migration.

MUC1, E-cadherin and $\beta$-catenin have all been implicated in pancreatic cancer development (20-23). The loss of E-cadherin gene expression can cause the dysfunction of the cell-cell junction to trigger tumor metastasis. Kondo et al (24) reported that decreased MUC1 expression led to increased E-cadherin-mediated cell-cell adhesion, suggesting that MUC1 competes with E-cadherin for binding to $\beta$-catenin. This prediction was confirmed in a previous study using MUC1-transfected HEK293T and HeLa cells (25). Some studies have reported that cancer patients with aberrant MUC1, E-cadherin or $\beta$-catenin expression have a worse prognosis when compared to similarly matched patients (26). It is possible that alterations in $\beta$-catenin and E-cadherin protein expression and localization are a long-term adaptive cellular reaction to MUC1 siRNA.

Those results supported the hypothesis that KL-6/MUC1 plays a complex role in the regulation of pancreatic cancer metastasis by directly or indirectly, regulating E-cadherin and $\beta$-catenin protein expression. 


\section{References}

1. Ellison LF and Wilkins K: An update on cancer survival. Health Rep 21: 55-60, 2010.

2. Hollingsworth MA and Swanson BJ: Mucins in cancer: protection and control of the cell surface. Nat Rev Cancer 4: 45-60, 2004.

3. Kohno N, Akiyama M, Kyoizumi S, Hakoda M, Kobuke K and Yamakido M: Detection of soluble tumor-associated antigens in sera and effusions using novel monoclonal antibodies, KL-3 and KL-6, against lung adenocarcinoma. Jpn J Clin Oncol 18: 203-216, 1988

4. Kohno N, Inoue Y, Hamada H, et al: Difference in serodiagnostic values among KL-6-associated mucins classified as cluster 9. Int J Cancer 57 (Suppl 8): 81-83, 1994.

5. Tang W, Inagaki Y, Kokudo N, et al: KL-6 mucin expression in carcinoma of the ampulla of Vater: association with cancer progression. World J Gastroenterol 11: 5450-5454, 2005.

6. Guo Q, Tang W, Inagaki Y, et al: Clinical significance of subcellular localization of KL-6 mucin in primary colorectal adenocarcinoma and metastatic tissues. World J Gastroenterol 12: 54-59, 2006.

7. Xu HL, Inagaki Y, Tang W, et al: Elevation of serum KL-6 mucin levels in patients with cholangiocarcinoma. Hepatogastroenterology 55: 2000-2004, 2008.

8. Inagaki $\mathrm{Y}, \mathrm{Xu} \mathrm{HL}, \mathrm{Nakata} \mathrm{M}$, et al: Clinicopathology of sialomucin: MUC1, particularly KL-6 mucin, in gastrointestinal, hepatic and pancreatic cancers. BioScience Trends 3: 220-232, 2009.

9. $\mathrm{Xu} \mathrm{HL}$, Inagaki Y, Wang FS, Nakata M, Kokudo N and Tang W: Effect of benzyl- $N$-acetyl- $\alpha$-galactosaminide on KL-6 mucin expression and invasive properties of a human pancreatic carcinoma cell line. Drug Discov Ther 2: 282-285, 2008.

10. Lustig B and Behrens J: The Wnt signaling pathway and its role in tumor development. J Cancer Res Clin Oncol 129: 199-221, 2003.

11. Ohno T, Aihara R, Kamiyama Y, Mochiki E, Asao T and Kuwano H: Prognostic significance of combined expression of MUC1 and adhesion molecules in advanced gastric cancer. Eur J Cancer 42: 256-263, 2006.

12. Baldus SE, Monig SP, Huxel S, et al: MUC1 and nuclear beta-catenin are coexpressed at the invasion front of colorectal carcinomas and are both correlated with tumor prognosis. Clin Cancer Res 10: 2790-2796, 2004.

13. Japan Pancreas Society: General Rules for the Study of Pancreatic Cancer. 5th edition. Kanehara-Shuppan, Tokyo, 2002.

14. Sobin LH and Wittekind C: TNM classification of malignant tumors. 5 edition. Wiley-Liss, New York, 1997.
15. Ohashi K, Murakami Y and Maruyama M: Four cases of mucin producing cancer of the pancreas on specific findings of the papilla of Vater. Prog Dig Endoscopy 20: 348-351, 1982 .

16. Zamora C, Sahel J, Cantu DG, et al: Intraductal papillary or mucinous tumors (IPMT) of the pancreas: report of a case series and review of the literature. Am J Gastroenterol 96: 1441-1447, 2001.

17. Said R, O'Reilly EM, Blumgart L, Shia J and Abou-Alfa GK: Pancreatic islet cell carcinoma presenting with concurrent Cushing's and Zollinger-Ellison syndromes: case series and literature review. Eur J Gastroenterol Hepatol 22: 246-252, 2010.

18. Li Y, Ren J, Yu W, et al: The epidermal growth factor receptor regulates interaction of the human DF3/MUC1 carcinoma antigen with $\mathrm{c}-\mathrm{Src}$ and beta-catenin. J Biol Chem 276: 35239-35242, 2001.

19. Li Y, Bharti A, Chen D, Gong J and Kufe D: Interaction of glycogen synthase kinase 3 beta with the DF3/MUC1 carcinomaassociated antigen and beta-catenin. Mol Cell Biol 18: 7216-7224, 1998.

20. Perl AK, Wilgenbus P, Dahl U, Semb H and Christofori G: A causal role for E-cadherin in the transition from adenoma to carcinoma. Nature 392: 190-193, 1998.

21. Schroeder JA, Adriance MC, Thompson MC, Camenish TD and Gendler SJ: MUC1 alters $\beta$-catenin-dependent tumor formation and promotes cellular invasion. Oncogene 22 : 1324-1332, 2003.

22. Wesseling J, van der Valk SW, Vos HL, Sonnenberg A and Hilkens J: Episialin (MUC1) overexpression inhibits integrinmediated cell adhesion to extracellular matrix components. J Cell Biol 129: 255-265, 1995.

23. Wen Y, Caffrey TC, Wheelock MJ, Johnson KR and Hollingsworth MA: Nuclear association of the cytoplasmic tail of MUC1 and $\beta$-catenin. J Biol Chem 39: 38029-38039, 2003.

24. Kondo K, Kondo N, Yokoyama A and Hiwada K: Decreased MUC1 expression induces E-cadherin-mediated cell adhesion of breast cancer cell lines. Cancer Res 58: $2014-$ 2019, 1998.

25. Li Y, Bharti A, Chen D, Gong J and Kufe D: Interaction of glycogen synthase kinase $3 \beta$ with the DF3/MUC1 carcinomaassociated antigen and $\beta$-catenin. Mol Cell Biol 18: 7216-7224, 1998.

26. Kimura T, Tanaka S, Haruma K, Sumii K, Kajiyama G, Shimamoto F and Kohno N: Clinical significance of MUC1 and E-cadherin expression, cellular proliferation, and angiogenesis at the deepest invasive portion of colorectal cancer. Int J Oncol 16: 55-64, 2000. 